\title{
Flow blockage in a transonic axial flow compressor: simulation analysis under distorted conditions
}

\author{
G. Srinivas ${ }^{1 *}$, K. Raghunandana ${ }^{2}$ B. Satish Shenoy ${ }^{3}$ \\ ${ }^{1}$ Assistant Professor, Dept of Aero \& Auto Engg, Manipal Institute of Technology, Manipal University, Manipal, Udupi, Karnataka. \\ ${ }^{2}$ Professor, Dept of Mechatronic Engg, Manipal Institute of Technology, Manipal University, Manipal, Udupi, Karnataka. \\ ${ }^{3}$ Professor, Dept of Aero \& Auto Engg, Manipal Institute of Technology, Manipal University, Manipal, Udupi, Karnataka. \\ *Corresponding author E-mail:srinivasle06@gmail.com
}

\begin{abstract}
Today the aircraft industry is looking for faster and safer engines for both civil and military applications. The performance of all different types of air breathing engines depends on the amount of mass flow rate of air entering and hot gas ejecting out from the engine. Thrust is the key role for any engine performance. To achieve more thrust all the turbo machinery components like axial fan, axial flow compressor and axial flow turbine should function effectively. This paper is primarily dealing about one of the turbo machinery component, axial flow compressor performance where the study is more focused on flow blockage formation under distorted phenomena. The complete blade boundary layer formation and related flow numerical theory are discussed in detail, accordingly the boundary conditions were set to have better numerical simulations using ANSYS tool. To find the flow blockage formation suitable turbulence model was coded using the well know compressible equations. The flow blockage between the rotor and stator of the compressor stage was calculated and also validated with that of experimental data effectively. The flow simulation results also revealed that the performance parameters under the modern engine transonic speed from Mach 0.8 to 1.2 under the distorted conditions are better for aeromechanical features.
\end{abstract}

Index Terms: Compressor, blockage, distortion, simulation, analysis.

\section{Introduction}

In the recent years the importance of turbo machinery in aircraft industry has increased drastically. Especially the transonic aircraft engines are developing more. The present challenges in the aircraft transonic engines are to achieve greater thrust with minimum fuel usage. The greater thrust is possible only when all the turbo machinery components function effectively. The efficiency of the turbo machinery components again depends upon the good aero dynamical and mechanical aspects. This paper is focusing more on the transonic axial flow compressor performance related to the aerodynamics under the distorted phenomena.

Distortion is the condition where the huge turbulence formation takes place near the tip of the compressor blade due to unexpected design environment [1]. The unexpected design environment may be because of situations like sudden acceleration and deceleration of the aircraft, sudden pull ups and downs of the aircraft and rolling of the aircraft. Under these non-design environmental conditions, the stall in the compressor may fluctuate and form blockages which will eventually reduce the performance of the compressor.

A systematic literature was conducted on the compressor performance under the non-design environment conditions. The literature reveals that under transonic flow condition the calculation of flow blockage in the rotor blade are not much explored.

Smith [2] worked on the flow blockage sources in the axial flow compressor. Later Smith also proposed the new theory especially for the non-design environment of a multistage axial flow compressor using boundary layer calculations in the general flow conditions. The limitations of his work was that there are no calculations shown for the transonic flow conditions. Later Koch [3] developed blockage development in the low subsonic axial flow compressor. It was concluded that changing the blockage position in the blade span gives mere deflections in the flow field. Taking motivation from Smith and Koch the flow blockage was further studied by Khalid especially near the blade tip regions [4]. His research also focused on low speed axial flow compressor but he solved using Navier stokes equations under the compressible flow conditions. Kenneth L Suder [5] has completed the experimental simulations on the rotor flow blockage under transonic flow conditions using the laser anemometer. He also concluded that a high loaded and high core flow sections of the blade could not cover the full blockage of the blade. For this kind of non-design environmental flow conditions CFD simulations play a vital role. The cost of simulations are less and the accuracy of the simulations are also decent enough to identify the flow blockage.

This paper aims to calculate the distortion effect through blockage development in the compressor under the transonic flow condition using CFD simulations which results in a better stream line flow over the blade and greater thrust of the engine so as to increase the aeromechanical features. The results obtained from the numerical analysis revealed that performance parameters of the compressor matched well with that of experimental work done by Kenneth $\mathrm{L}$ Suder [5].

H.K. Ozturk et al[6] discussed the design of the stator blade in a modern high-pressure turbofan engine. The purpose of this paper is to show turbulence effects, without flow blockage, especially seal leakage, can be reduced by incorporating fins on the rotating component rotor blade. Shu Lin et al [7] studied active surge control system in a variable speed axial flow compressors. The compression system was equipped with a variable area throttle to 
find the surge flow parameters. In their work the flow description was limited only to single stage of an axial flow compressor.

NavidSharifi et al[8] in their study, prepared and validated a numerical model to investigate the flow pattern within a conventional thrust vector control (TVC). In this paper the numerical scheme was prepared on the basis of three dimensional assumptions and axisymmetric geometrical approaches. The scheme describes more emphasis on thermo-compressor design than flow behavior characteristics. Also it is observed that entire flow analysis was limited to steady flow thermal conditions.

Lingen Chen et al [9] studied about the design of an axial-flow compressor stage under subcritical Mach numbers with the objective of minimizing the aerodynamic losses and the weight of the stage. Aerodynamics as well as mechanical constraints are considered in the optimization solution. The prediction model for estimating the performance characteristics, such as efficiency, weight and stall margin, of the compressor stage was well presented. The model was not able to replicate results for transonic axial flow compressor under non design environmental conditions.

Xuemin Ye et al [10] implemented appropriate changes to the blade tip structure and found greater fan performance. The performance of the OB-84 axial fan with different grooved blade tips was simulated using Fluent. The effects of various tip structures on the flow field, losses distribution, and noise characteristics are investigated. Analysis of static structure and vibration characteristics is performed with ANSYS, finite element analysis module. The numerical simulations are limited to tip structure of the blade and couldn't show the complete fan annular section.

Chang Hao et al[11] initiated the comparative numerical studies to investigate the effects of blade sweep on inlet flow in axial compressor cascades. A series of swept and straight cascades was modelled in order to obtain a general understanding of the inlet flow field that is induced by sweep. A computational fluid dynamics (CFD) package was used to simulate the cascades and obtain the required three-dimensional (3D) flow parameters. The flow design conditions are limited to subsonic conditions.

Jin-Hyuk Kim et al [12] discussed about the transonic axial compressor with circumferential casing grooves optimization to improve operating stability. Numerical analysis was conducted by solving three-dimensional Reynolds-Averaged Navier-Stokes (RANS) equations using the shear stress transport turbulence model. An optimization process performed was based on a weighted-average surrogate technique under steady flow conditions. Analysis was carried out with three design variables defining the tip clearance, blade tip angle, and depth of the grooves. The results were limited to steady flow conditions.

\section{Understanding the flow domain boundary layer of the compressor stage under distorted condition}

The axial flow compressor rotor and stator blade flow velocity vectors can be calculated from the traditional velocity triangles. The flow regions at inlet of the rotor is considered as section 1 and outlet of the rotor or inlet of the stator as section 2 and consecutive stator outlet section as 3 . The flow passing relatively over the rotor suction surface of the blade is termed as relative velocity and is denoted by $V$. The flow which covers completely both the suction and pressure surfaces of the rotor blade is termed as absolute velocity and is represented as $C$. The rotor shaft connects the turbine shaft to give the required design speed $(U)$. Flow perpendicular to the design speed gives the flow angles $(\alpha, \beta)$ and is shown in the Fig 1. After combining both the rotor and stator velocity triangles, the compressor stage velocity triangles along with the axial and tangential velocity components are obtained and is as shown in Fig 2. The aircraft engine works on Brayton cycle and it is used for calculation of compressor and turbine efficiencies. The P-V and T-S diagrams of Brayton cycle is shown in Fig 3.
From the velocity triangle the efficiency of the axial flow compressor is calculated using Equation (1).

$$
\eta=\frac{W}{Q}=\frac{W_{34}-W_{12}}{Q_{23}}=\frac{C_{P}\left(T_{3}-T_{4}\right)-C_{p}\left(T_{2}-T_{1}\right)}{C_{p}\left(T_{3}-T_{2}\right)}
$$

Where $W$ is the work done by the compressor, $Q$ is the heat supplied to the system $C_{p}$ is the specific heat at constant pressure of the air (The value of $C_{p}$ for air $=1.005 \mathrm{KJ} / \mathrm{Kg}$. K) and the temperatures $T_{1}$ to $T_{4}$ values are taken from the turbofan engine Brayton cycle as shown in Fig 3. However the full numerical boundary conditions for the study of blockage in the compressor rotor are further discussed in the subsequent sections of this research paper.

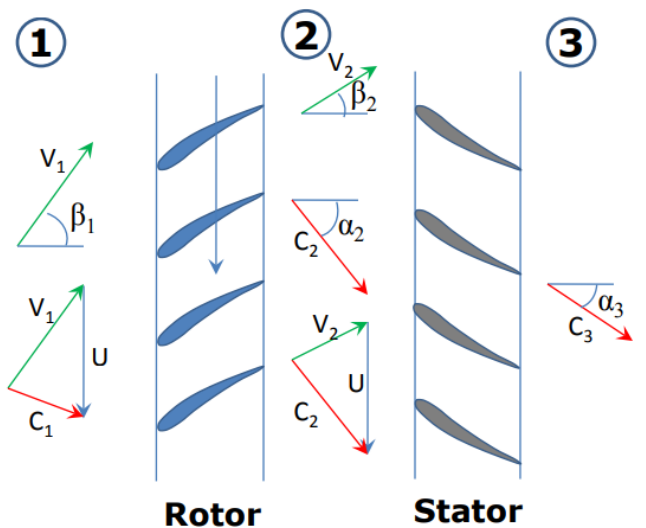

Figure 1: Representation of rotor and stator velocity triangles

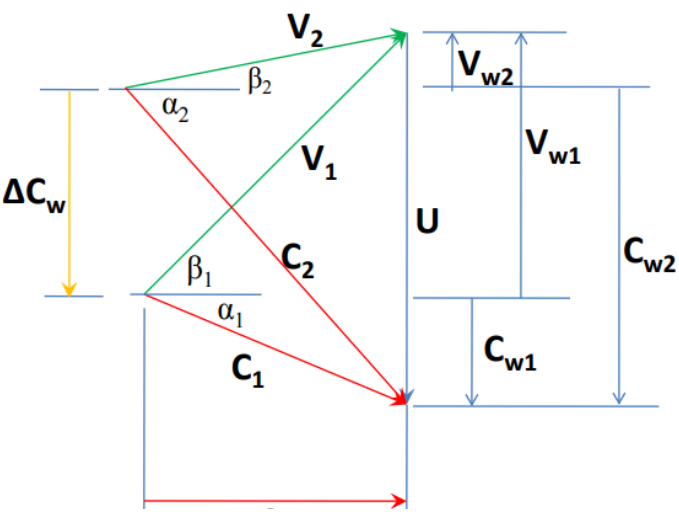

Figure 2: Representation of stage velocity triangles
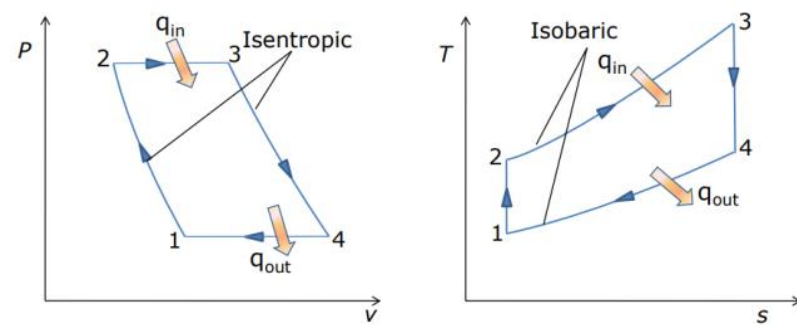

Figure 3: Representation of stage thermodynamic cycles The primary source for flow blockage is the bubble formation at tip region of the rotor blade, and it further progresses as a vortex (i.e. vortex rounds) development. Eliminating the vortex formation in any rotor passage is not possible but using suitable CFD flow turbulence solver we can minimize the vortex effect. From the well-known compressible flow equations we can calculate the boundary layer calculations across the rotor blade. The easy way to compute the flow blockage across the stages is by using the unsteady, non-uniform, viscid, compressible flow equations. Another possible way of minimizing the vortex growth is basically understanding the blockage regions. The blockage regions can be computed using the basic fluid dynamics compressible flow boundary layer equations. To know the boundary layer flow across the rotor, it is essential to know the displacement thickness $\left(\delta^{*}\right)$ parameter and it can be estimated from the boundary layer thickness $(\delta)$ from the Equation (2).The compressor blade tip 
vortex momentum thickness $(\theta)$ is evaluated from the Equation (3), where $U_{\infty}$ is characteristic transonic flow velocity, u is free stream velocity, $d y$ is the distance of the elemental boundary layer thickness in y-direction [13-14].

$$
\begin{gathered}
\delta^{*}=\int_{0}^{\delta}\left(1-\frac{u}{U_{\infty}}\right) d y \\
\theta=\int_{0}^{\delta} \frac{u}{U_{\infty}}\left(1-\frac{u}{U_{\infty}}\right) d y
\end{gathered}
$$

Vortex generating at the tip region of the rotor blades can be redesigned mathematically by free vortex design method. Vortex of the flow domain depends upon the angular velocity of stream and radius of the blade. The vortex formation further leads to energy dissipation on the flow which spoils the compressor stall in the performance curve. Therefore calculating the energy changes before and after the rotor flow is essential to re-design the vortex phenomena in the control volume. Therefore the energy dissipation can be measured as shown in Equation (4) by change of stagnation enthalpy $\left(\Delta h_{o}\right)$ which is the product of blade speed $(U)$ and the difference in tangential component of absolute velocity $\left(\Delta C_{\theta}\right)$. Further the blade speed can be expressed in terms of angular velocity and radius of the blade as shown in Equation (5). From the equation it is understood that for a given rotational speed the $r$. $\Delta C_{\theta}$ must be constant.

$$
\begin{gathered}
\Delta \mathrm{h}_{\mathrm{o}}=\mathrm{U} . \Delta \mathrm{C}_{\theta} \\
\Delta \mathrm{h}_{\mathrm{o}}=\Omega \text {. . r. } \Delta \mathrm{C}_{\theta}
\end{gathered}
$$

Maintaining the low strength vortex formation is quite difficult task under the transonic speed (Mach number range from 0.8 to 1.2). To achieve this, calculating the blockage of the flow and minimizing the flow vortex is the only alternative method in the off-design technique. The tip vortex formation further forms as blockage in the flow field and this blockage can be defined in several ways. One of the well-known definition given by Kenneth L. Suder [5] is that it is the effective reduction in the flow area and is denoted by ' $\mathrm{B}$ ' shown in the Equation (6). Equation (7) represents the mathematics representation of blockage ' $\mathrm{B}$ ', Where $\mathrm{A}$ is the area of rotor blade. The vortex formation is caused only because of the top region of the rotor tip. The tip region velocity $(u)$ and density $(\rho)$ of the blade is measured using the Equation (8). The flow in the control volume is considered to be a compressible and viscid flow. Hence the density of air keeps changing as the aircraft operates under non-design environmental conditions. Therefore the static pressure throughout the stage monotonically increases and also it is noted that the downstream of the energy dissipating in the stator blade is because of no contribution from the stator. Only the kinetic energy of the flow increases due to the consecutive rotor blade. More blockage formation happens at the blade pressure side through the downstream of the flow. Therefore the down streams of the boundary layer thickness is needed to be estimated under both unsteady and non-uniform conditions. The exact area of flow blockage is further demonstrated through cut-off region at rotor blade tip. Equation (9) describes the flow in the downstream condition to calculate the flow description using boundary layer thickness under non-uniform and unsteady flow region. Equation (10) helps in identifying the blockage defect cut-off region and also to enhance the performance of the compressor stage. This paper provides the full understanding of flow blockage in rotor blade under the real viscid conditions. Therefore the blade speed ratio defined with respect to the viscid and boundary layer thickness as shown in the Equation (11). The total temperature and total pressure across the stage blockage are evaluated effectively using the Navier-stokes equation [13].

Blockage $(\mathrm{B})=[1-($ Effective Area of the rotor blade /

$$
\begin{gathered}
\text { Blade Geometric area })] \\
\mathrm{B}=1-\frac{\left(A-\int \delta^{*} d r\right)}{A} \\
\delta^{*} d r=\int_{\theta=0}^{2 \pi / n}\left(1-\frac{\rho u}{\rho u_{\text {viscid }}}\right) r d \theta
\end{gathered}
$$

$$
\begin{gathered}
\delta_{\text {downstream }}^{*}(r)=\int_{\theta=0}^{2 \prod / n}\left(1-\frac{T_{\text {viscid }} . u}{T_{\text {visid }}}\right) r d \theta \\
\text { Vortex region defect where: }\left|\frac{\partial u}{\partial r}\right|+\left|\frac{\partial u}{\partial(r \theta)}\right| \geq(\text { cutoff }) \\
\frac{U}{U_{\text {viscid }}}=\left(\frac{y}{\delta}\right)^{n}
\end{gathered}
$$

Computing the accurate numerical simulations through CFD tool is not a simple task, especially for flow simulation of rotating components like fan, compressor and turbine. These requires special solvers for solving the specified boundary conditions. Finding the compressor stage flow blockage through CFD tool requires appropriate geometrical model, error less mesh domain and high computing facility to run the simulations. However with the advanced high computational facility and through updated turbulence solvers it is possible to enhance compressor flow simulation performance. The modern computational solvers and recent trends in axial flow compressor under transonic flow conditions are well discussed by Srinivas [15]. Using the above cited equations under the transonic flow conditions the complete flow domain boundary conditions are calculated. To have a better flow simulation, rotor 37 blade data [16] is considered to produce the geometrical model and the structured mesh is created. For the successful completion of the numerical simulations of rotor to stator flow blockage CFD tool is used and is described in the subsequent sections.

\section{Geometrical model for flow blockage around the rotor to stator blades}

\begin{tabular}{|c|c|}
\hline Description & Designed Values \\
\hline Number of rotor blades & 36 \\
\hline Number of stator blades & 37 \\
\hline Distance from axis of rotation to blade hub & $0.1778 \mathrm{~m}$ (7 inches) \\
\hline Rotational speed & $17188.7(\mathrm{rpm})$ \\
\hline Blade aspect ratio & 1.19 \\
\hline Mach number operating conditions & $\begin{array}{l}0.8 \text { to } 1.2 \text { cases (Transonic } \\
\text { speed) }\end{array}$ \\
\hline
\end{tabular}

In order to investigate the flow blockage effects on the axial flow compressor stage(i.e. both rotor and stator blades) the NASA Rotor 37 test case data was used as the baseline geometry. To find out the distortion effects on rotor blade complete compressor stage is focused rather than the rotor blade alone. Complete rotor 37 geometry data and the related performance data are reported in [16]. Full axial flow compressor design specifications are tabulated in Table 1.

Table 1: Axial Flow Compressor Design Specifications [16]

The axial flow compressor stage consists of 36 rotor blades and 37 stator blades. In order to reduce the numerical simulation time effort, only one stage of the full axial flow compressor geometry was modelled. The numerical simulation geometry model consist of one rotor blade and one stator blade. This leads to blade pitch ratio at the interface which is equal to 1.2 instead of 1.278 of the real geometry and also twist angles of 40 degrees for the rotor and 30 degrees for the stator blade. The computational grids were generated using ANSYS tool [17]. The computational geometry model and grid plots are shown in Fig 4-8.In the computational domain to have the good quality mesh elements with no errors Y+ axis boundary layer values must be maintained as high as possible. In order to increase the $\mathrm{Y}+$ boundary layer around the blades, prism layers were added on the surface of the blade using tetrahedral mesh elements. The grid independence variation was also plotted for better consistency results and is shown in Fig 18. The Y+ scalable wall function values plotted with the different mesh size values as shown in Fig 9. After successful completion of the grid independence it was identified that 2, 05,000 mesh element size was appropriate for the Mach speed from 0.8 to 1.2. To get high 
quality simulations on the stage, prism type mesh elements with 12 layers was maintained with a grid point accuracy size of 25 micrometer. The selection of first grid point position for the speed choice is reported in reference [18].
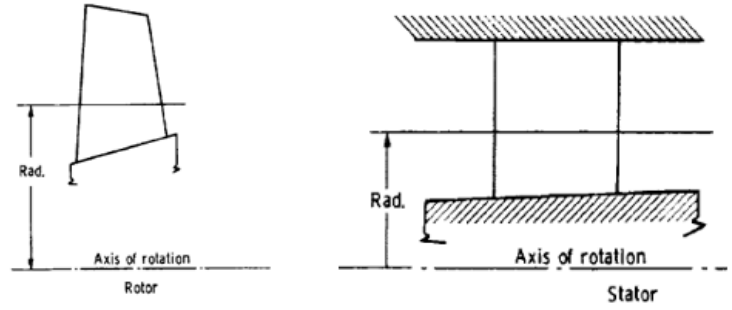

Figure 4: Blade basic coordinates representation [16]

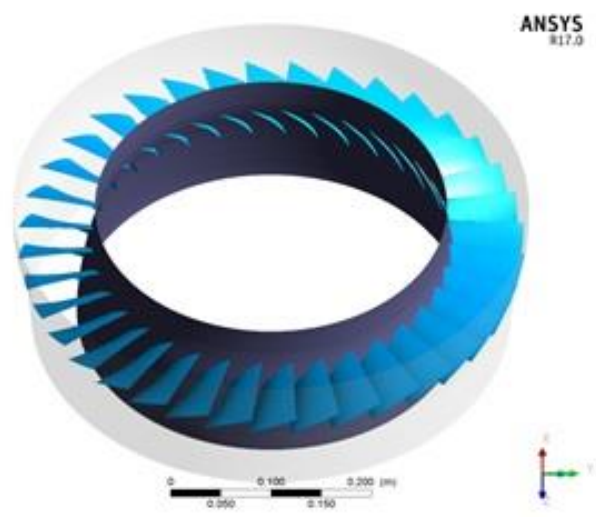

Figure 5: ANYS Geometrical model of Rotor 36 blades

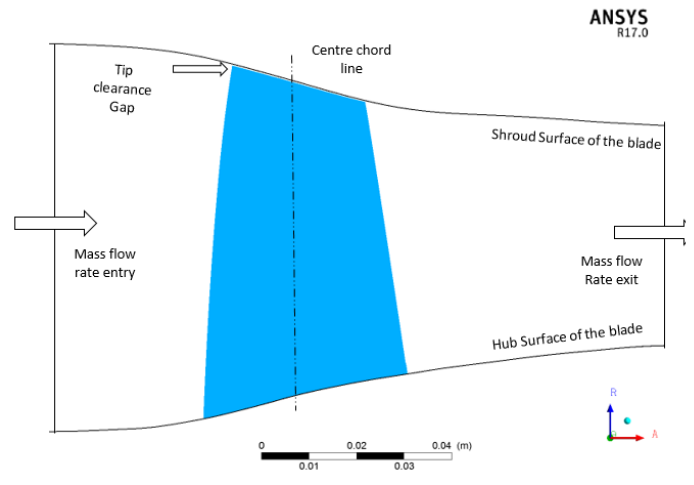

Figure 6: Geometrical details of rotor blade in ANSYS

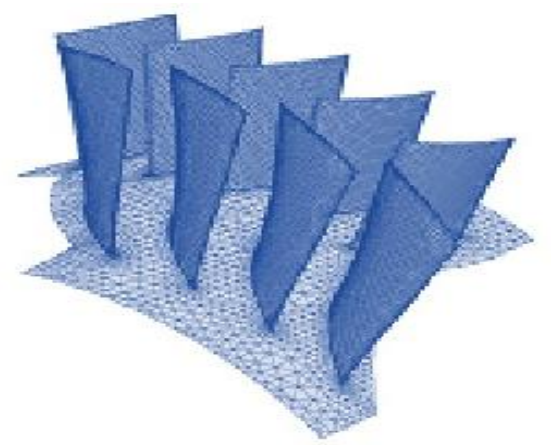

Figure 7: Representation of mesh elements on compressor stages

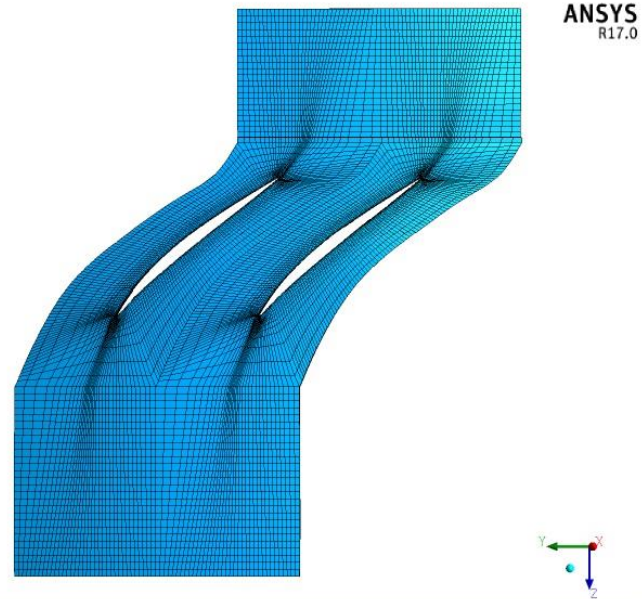

Figure 8: Representation of mesh elements near the leading edge and trailing edge in ANSYS

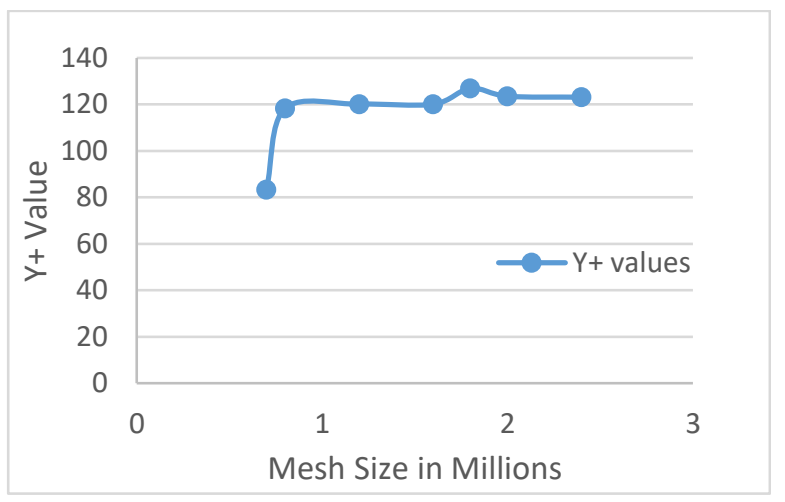

Figure 9: Representation of $\mathrm{Y}+$ values for model $100 \%$ design speed

\section{Stage flow blockage numerical simulation model}

After successful completion of the rotor 37 stage geometrical model, error free mesh is created in the flow domain. The flow domain is also defined with flow inlet and flow outlet boundary conditions. The flow simulations is conducted by using the ANSYS CFD tool [19]. The main function of ANSYS CFD code is to solve the RANS (Reynolds Average Navier Stokes) equations by using finite volume technique. To resolve the turbulence effect over the stage the additive correction multigrid approach was used. To calculate the distortion effects on rotor blade while giving the flow boundary conditions algebraic multigrid method was chosen. In the discrete finite volume equations third order high resolution scheme was implemented to see the distorted phenomena in the simulation. The CFD simulations were performed under clean offdesign environment with steady multiple frame of reference in order to see the disturbances in rotor and stator blades. A mixing plane approach was incorporated at the rotor and stator interface to see the losses around the blade hub to tip geometry including the suction and pressure surface of the blade profiles and it was adopted for all the simulations. However the blade plane position was adjusted according to the boundary conditions from Mach 0.8 to 1.2 type transonic based flow by giving the rotor and stator pitch ratio.

\section{Rotor flow blockage turbulence model}

To find the flow blockage in both the low and high speed compressor stage Kenneth L Suder [5] used a two color fringe type laser anemometer system to acquire the axial and tangential velocity components. But modern existing CFD turbulence models brings up high quality simulations that too under transonic speed 
conditions. In this research workfor the compressor stage simulations, ANSYS CFD turbulence model was used to solve the flow domain boundary conditions. To find out distortion effect under the transonic flow condition on the compressor stage the eddy-viscosity RANS two equation k-e model was used. The theory of scalable wall function methodology [20]was considered in the analysis. According to the analytical scalable wall function approach an improved turbulent velocity scale $\tilde{u}_{\tau}$ is used. This value is generally dependable on the turbulent model kinetic energy at blade suction side or pressure side wall node $k_{p}$.

$$
\tilde{u}_{\tau}=c_{\mu}^{1 / 4} k_{p}^{1 / 2}
$$

As a result an altered $\mathrm{y}+$ based on $\tilde{u}_{\tau}$ can be evaluated using

$$
\tilde{y}^{+}=\tilde{u}_{\tau} y / v
$$

The logic behind implementing the scalable wall function method is to minimize the numerical $\mathrm{y}+$ number utilized in logarithmic formulation. For the unsteady and non-uniform flow generally the scalable wall function $\mathrm{Y}+$ value should not be more than 15 [20]. Therefore in this simulation work the value of $\mathrm{Y}+$ scalable wall function is maintained as 10.06 which is reasonable for all real flow boundary conditions. To calculate the flow near wall distortion effect in the compressor stage, the k-e model needs to be changed. The wall functions for transonic flow are defined in the above two Equations (12-13).By incorporating the above said $\mathrm{Y}+$ scalable wall function, the logarithmic wall profile will exist throughout the stage flow domain and also, flow turbulence effect can simulate effectively under the given set of boundary conditions.

\section{Rotor flow blockage boundary conditions}

To analyze the flow distortion blockage influence on axial flow compressor at the inlet condition it is required to impose the appropriate flow angle, total pressure and total temperature. Axial flow compressor stage inlet conditions are fixed at inlet total temperature $\left(\mathrm{T}_{01}\right)=288.15 \mathrm{~K}$ and inlet total pressure $\left(\mathrm{P}_{01}\right)$ $=101,325 \mathrm{~Pa}$ [14]. Work carried out in this paper is to simulate the flow blockage effect of transonic compressor stage. The relevant data under 100 percent design speed line near the chocked conditions were chosen from reference [16]. Hence for the flow domain inlet relative static pressure ( $\mathrm{P}_{\text {rel.static }}$ ) was set from zero Pascal.

This value was maintained until it reaches the near stall conditions since the flow convergence criteria of 0.000001 is met only at the near stall condition. Simulation was also carried out for different mass flow rates under the same distorted conditions. The flow behavior is restricted under transonic conditions from the Mach speed ranging from 0.8 to 1.2 . Only one complete stage of axial flow compressor is simulated for all the boundary conditions. For effective visualization of the vortex, angular velocity value is also incorporated in the stage boundary conditions as mentioned in the 100 percent design speed line of reference [16]. In order to the see the effect of blockage under non-design environment conditions the designed speed was maintained for all the cases as $17,188.7 \mathrm{rpm}$ [16].

\section{Simulation results and discussion}

After setting the stage distorted boundary conditions, the flow simulations were performed until the solution converges. The simulated flow results were later validated with the experimental data given in reference [16]which is for transonic high loaded compressor (Mach speed range from 0.8 to 1.2). The compressor stage flow disturbances were calculated by using k-e turbulence model, especially near the tip regions where more leakage is possible when the fluid is moving from rotor to stator blade. For this reason the value of $\mathrm{k}_{\mathrm{p}}=1 \mu \mathrm{m}$ was imposed, which is aerodynamically very smooth condition $\left(\mathrm{Re}_{\mathrm{k}}=25\right)$.It is observed that the error between the numerical simulations to the experimental data was very much minimum and was in acceptable range. The total pressure ratio error noticed between numerical results to experimental data was $\pm 2.25 \%$ and is as shown in Fig 16. Similarly, the adiabatic efficiency was also calculated and \pm 1.25 percentage error was observed as shown in Fig 17. The mass flow rate of the compressor stage matched well with the experimental data with an error of $1.12 \%$ and is as shown in Fig 19.In the work carried out under transonic flow condition the validating points are taken from 100 percent design speed line analysis [16].

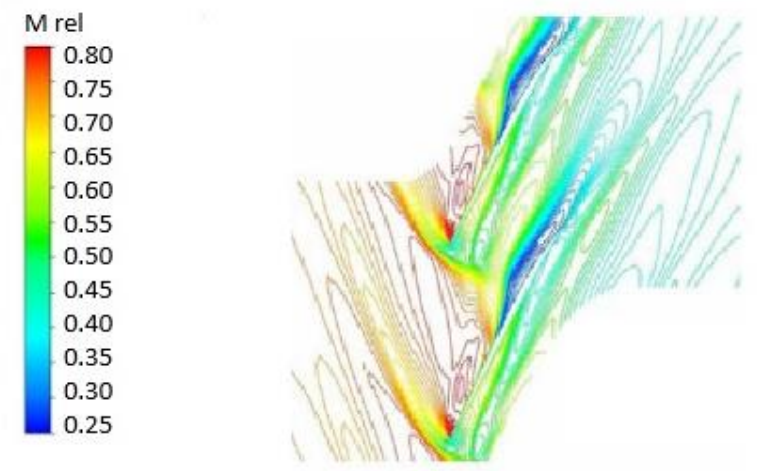

Figure 10: Mach number distribution on stage at Mach 0.8

The aerodynamic behavior and flow turbulence effects at different flow conditions on the rotor blade casing are also analyzed to see the distorted effects.

In this numerical analysis quite interesting points are noticed at the boundary layer of the rotor blade and flow interaction between the fluid structures. In the case of Mach number 0.8 the shock is more detached and Mach relative flow speed keeps on diminishing from leading edge to trailing edge of rotor blade as shown in Fig 10. At Mach number 1.0 flow condition, the tip vortex was found very strong at the leading edge of the rotor as shown in Fig 11. It is found that the reduction in flow separation after the shock, leads to low velocity flow across the blade at Mach number 1.2 and is shown in Fig. 12.

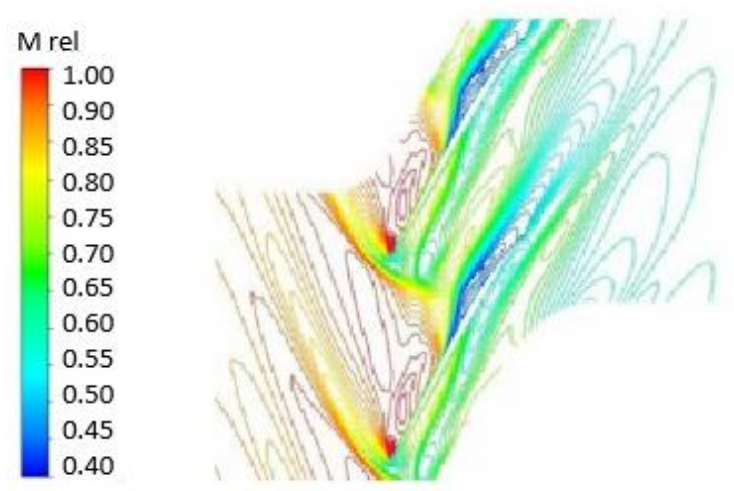

Figure 11: Mach number distribution on stage at Mach 1.0

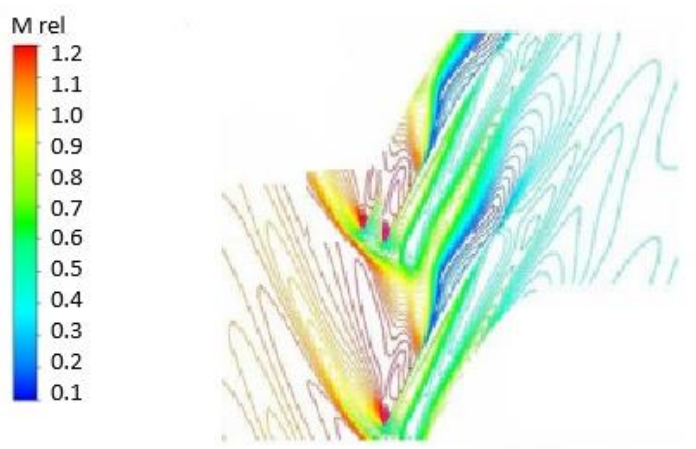

Figure 12: Mach number distribution on stage at Mach 1.2 
The flow blockage performance is further analyzed by conducting the CFD simulations with different designed air mass flow rate of aircraft engine. Kenneth L Suder [5] also analyzed experimentally the compressor stage blockage effects at different mass flow rates of air. Fig 21 describes the total pressure ratio versus mass flow rate of the air passing on the stage. The numerical results and experimental results are well matched at the mass flow rate of 24 $\mathrm{kg} / \mathrm{sec}$ and could achieve maximum total pressure ratio as shown in Fig 21. Because of the blockage effect the transonic compressor stage simulation results are not meeting exactly with the experimental data. It is also noticed that after a mass flow rate of $24 \mathrm{~kg} / \mathrm{sec}$, the total pressure ratio could not achieve higher value due to the chocking effect.

Due to the distortion effect the blade tip generates air pockets which is generally termed as "flow blockage vortex rounds". This phenomena happens when the flow reaches from subsonic conditions (Mach number less than 0.8) to transonic conditions (Mach number 0.8 to 1.2). For better understanding of distortion effects, the stage simulation is also showed in the formation of three dimensional flow air pockets for all transonic flow conditions. Fig 13 clearly shows the formation of air pockets near the tip of the blade corners and it is also noticed that the velocity of these pockets are an average $216 \mathrm{~m} / \mathrm{sec}$. With the increase in flow condition at Mach number 1.0 it is observed that air pockets velocity increases to $225 \mathrm{~m} / \mathrm{sec}$ as shown in Fig 14. At Mach number 1.2 the velocity of air pockets is much higher than 355 $\mathrm{m} / \mathrm{sec}$ as shown in Fig 15.

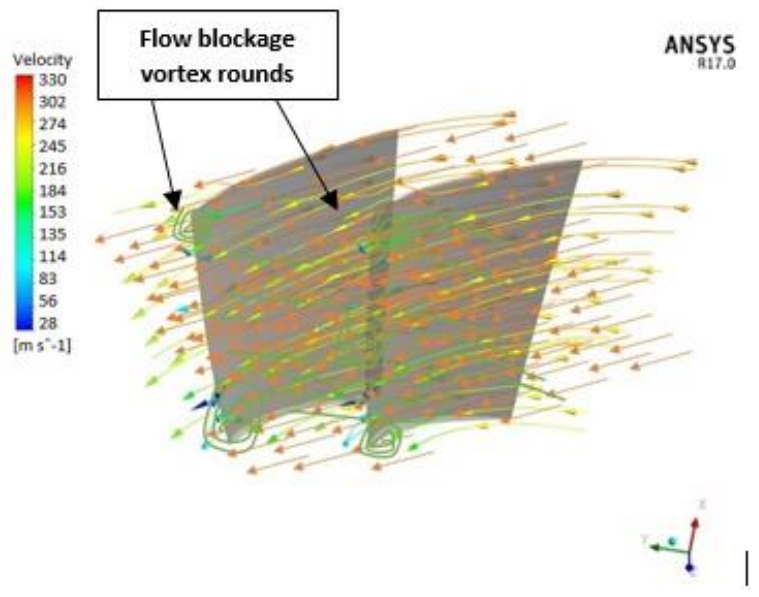

Figure 13: Vortex flow near the tip of the rotor blade at mach 0.8

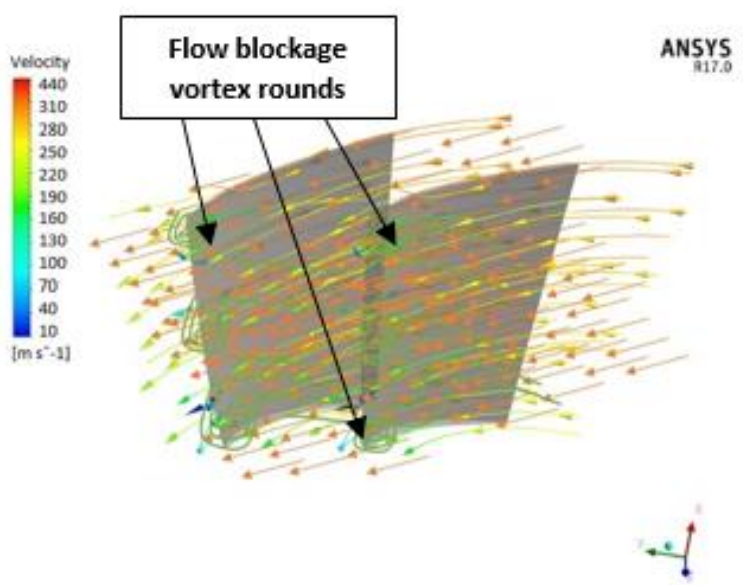

Figure 14: Vortex flow near the tip of the rotor blade at mach 1.0

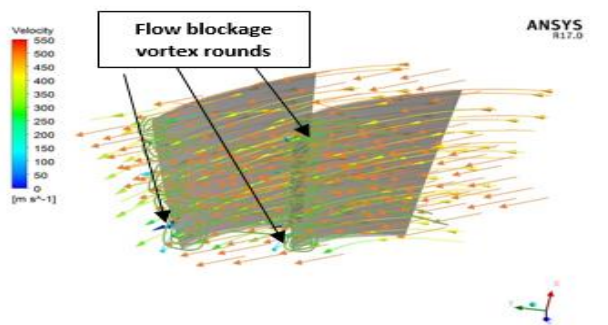

Figure 15: Vortex flow near the tip of the rotor blade at mach 1.2

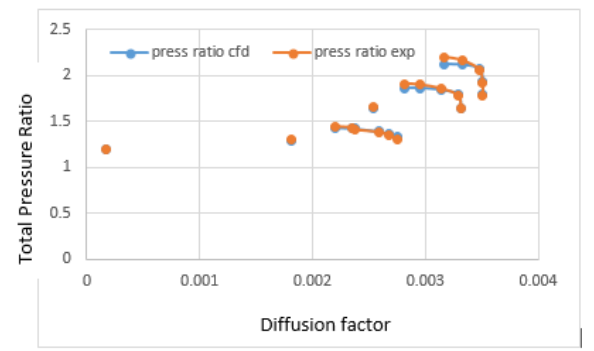

Figure 16: Compressor pressure ratio model validation [16]

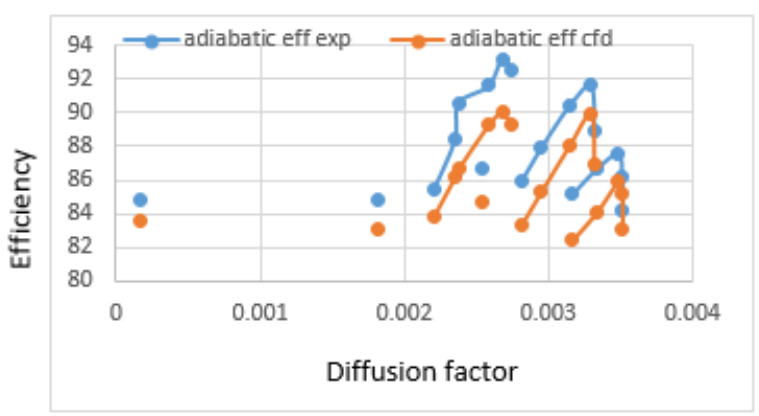

Figure 17: Adiabatic efficiency for stage 37, 100\% design speed

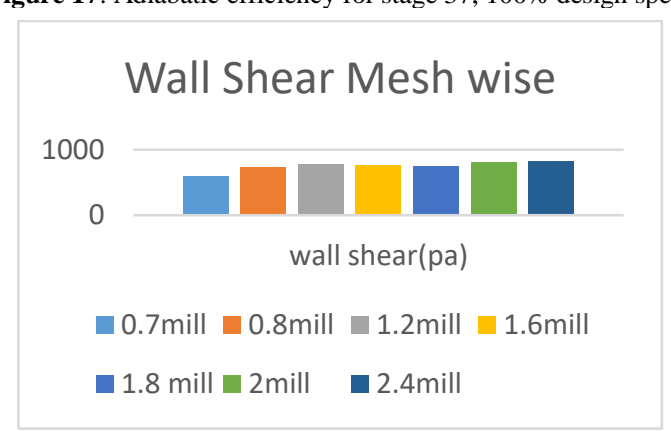

Figure 18: Grid independence check of stage 37, 100\% design speed

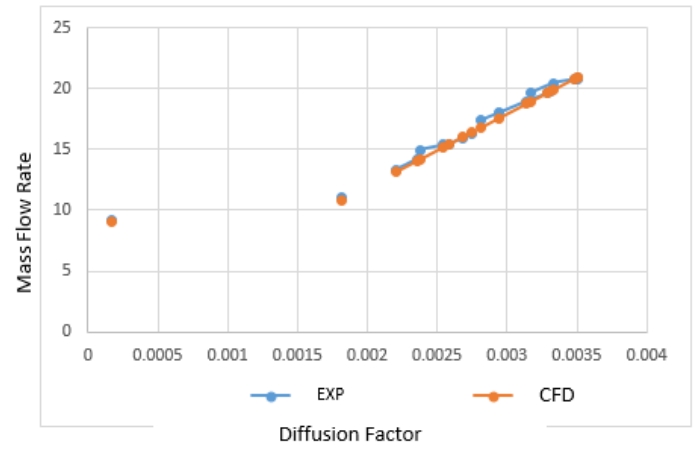

Figure 19: Mass flow rate of stage 37, 100\% design speed 


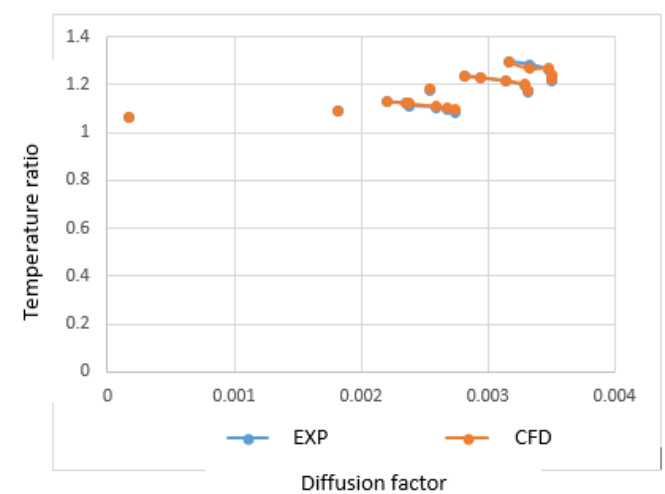

Figure 20: Temperature ratio of stage 37, 100\% design speed

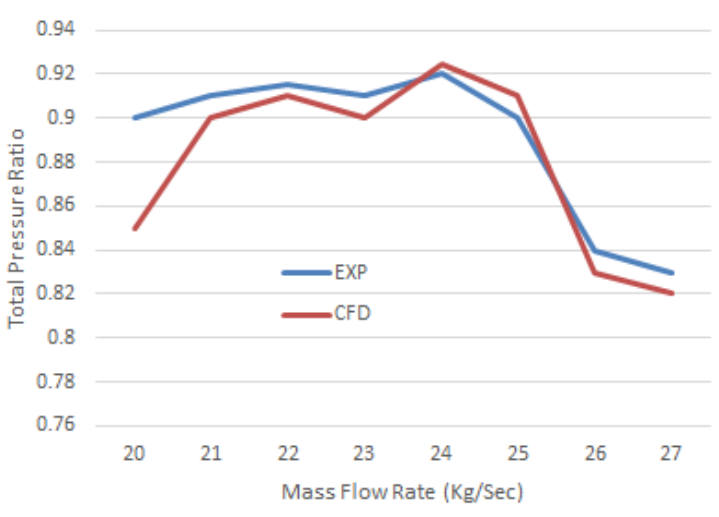

Figure 21: Comparison of total pressure ration with different mass flow rate of compressor stage [5]

\section{Conclusion}

The flow blockage numerical analysis of an axial flow compressor stage is accomplished by using NASA Rotor 37 and Stator 37 blade data, which was implemented by using ANSYS commercial CFD tool. To evaluate the distortion effect and overall performance of the axial flow compressor stage, geometrical model was created in CFD tool exactly like the realistic model as given in literature [16]. The compressor stage geometrical model was used to simulate the occurrence of distortion by incorporating different boundary conditions. From the numerical simulation of compressor stage it is observed that the blockage effect is more near the rotor tip and the mass rate of air also decreased at downstream of the flow domain. This blockage effect leads to reduce the performance of the compressor. Due to the sudden formation of flow vortex blockage the pressure ratio at the tip region further decreases and this effect was more at Mach speed 1.2 than at Mach speed 0.8 condition. Further it is observed that at Mach speed 1.0 the flow is having low vortex strength and the flow conditions are matching with the off-design and design experimental boundary conditions to maintain the greater thrust from the aircraft engine. In the numerical simulations, different unsteady and non-uniform flow combinations were attempted to see the flow effect on rotor and stator blade. Due to the development of high kinetic energy in the rotor blade, the flow distortion effect is more on the rotor blade than on stator blade. Through this numerical analysis it has been observed noticeably that, in the 100 percent speed line the distortion phenomena is more influencing near the tip axial chord of rotor blade. Distortion flow blockage effect was much higher at suction surface and even at the span wise direction of the rotor blade. Axial flow compressor flow blockage simulation analysis results can be used to estimate the pressure characteristics of the full rotor annulus in a new compressor design development.

\section{References}

[1] Mokelke H, "The Unsteady Response of an Axial Flow Compressor with a Distorted Inlet Flow", ministry of defense (procurement executive) aeronautical research council current papers, (1970).

[2] Smith JLH, "Casing Boundary Layers in Multistage Axial-Flow Compressors", Flow Research on Blading, (1970), pp.275-304

[3] Koch CC, "Stalling Pressure Rise Capability of Axial Flow Compressor Stages", ASME Journal of Engineering for Power, Vol.103, (1981), pp.645-656.

[4] Khalid S, "The Effects of Tip Clearance on Axial Compressor Pressure Rise, Ph.D. Dissertation, Massachusetts Institute of Technology, (1994).

[5] Suder KL, "Blockage development in a transonic, axial compressor rotor", ASME International Gas Turbine and Aeroengine Congress and Exhibition (pp. V001T03A059-V001T03A059), (1997).

[6] Öztürk HK, Turner AB, Childs PRN \& Bayley FJ, "Stator well flows in axial compressors", International journal of heat and fluid flow, Vol.21, No.6, (2000), pp.710-716.

[7] Lin S, Yang C, Wu P \& Song Z, "Active surge control for variable speed axial compressors", ISA transactions, Vol.53, No.5,(2014), pp.1389-1395.

[8] Sharifi N \& Boroomand M, "An investigation of thermocompressor design by analysis and experiment: Part 1. Validation of the numerical method", Energy conversion and management, Vol.69, (2013), pp.217-227.

[9] Chen L, Sun F \& Wu C, "Optimum design of a subsonic axial-flow compressor stage”, Applied energy, Vol.80, No.2,(2005), pp.187195.

[10] Ye X, Li P, Li C \& Ding X, "Numerical investigation of blade tip grooving effect on performance and dynamics of an axial flow fan", Energy, Vol.82, (2015), pp.556-569.

[11] Chang H, Zhu F, Jin D \& Gui X, "Effect of blade sweep on inlet flow in axial compressor cascades", Chinese Journal of Aeronautics, Vol.28, No.1,(2015), pp.103-111.

[12] Kim JH, Choi KJ \& Kim KY, “Aerodynamic analysis and optimization of a transonic axial compressor with casing grooves to improve operating stability", Aerospace Science and Technology, Vol.29, (2013), pp.81-91.

[13] Bansal RK, "A Text Book of Fluid Mechanics and Hydraulic Machines”,(2015).

[14] Roy B, "Aircraft Propulsion-Science of making thrust to fly", Published by Elsevier, (2008).

[15] Sriniva G, Raghunandana K \& Satish shenoy B, "Recent developments of axial flow compressor under transonic flow conditions", IOP conference series: Material Science and Engineering, (2017).

[16] Reid L \& Moore RD, "Design and overall performance of four highly-loaded, high-speed inlet stages for an advanced highpressureratio core compressor", NASA TP, (1978).

[17] ANSYS ICEM CFD 17.0, 2017, User Manual.

[18] Cadorin M, Morini M \& Pinelli M, "Numerical Analyses of High Reynolds Number Flow of High Pressure Fuel Gas Through Rough Pipes", International Journal of Hydrogen Energy, Vol.35, (2010), pp. 7568-7579

[19] ANSYS CFX 11.0,User Manual.2007.

[20] Apsley D, "CFD Calculation of Turbulent Flow with Arbitrary Wall Roughness", Flow, Turbulence and Combustion, (2007), pp. 153-175. 